\title{
Impacts biologiques des pollutions sur un ruisseau périurbain : Le Peugue (Gironde)
}

\author{
par Franz Gayet, Michel Coste, Jean-François Dubernet, Alain Dutartre \\ Cemagref, Division Qualité des Eaux
}

\section{I $\square$ PRÉSENTATION DU SITE}

\subsection{Géographie}

Le ruisseau du Peugue prend sa source à Romainville sur la commune de Pessac. Il s'écoule selon une pente faible en direction SO-NE dans sa partie amont, puis O-E dans sa partie aval où il va se jeter dans la Garonne. Il traverse consécutivement les communes de Pessac et Mérignac où il est finalement canalisé (du Burck à la Garonne).

Ce ruisseau est alimenté essentiellement par les rejets pluviaux des lotissements et des pavillons avoisinants (le bassin versant est de type semi-urbain et draine une zone de 1212 hectares). L'urbanisation augmente de façon sensible dès que l'on dépasse la rocade, en se dirigeant vers le centre de Bordeaux.
Le Peugue est un ruisseau recalibré, comprenant sur son parcours un bassin d'étalement (site des Fauvettes) et un bassin de retenue (bassin du Bourgailh), tous deux mis en place pour la lutte contre les inondations.

\subsection{Caractéristiques du ruisseau}

Il existe une piste d'exploitation privée, le long du cours du Peugue, dont l'usage s'est étendu aux habitants des environs. Ce cours d'eau est devenu un lieu de passage et de promenade intensifs, dont l'accès piétonnier est aisé.

On peut remarquer à certains endroits des amoncellements de déchets tels que bouteilles en verre, sacs en plastique, pneus, paquets de cigarettes, etc.

On remarque une flore aquatique peu diversifiée et peu abondante, même si l'on ne se trouve pas encore dans la saison végétative.

\section{Biological impacts of pollution on a suburban little stream : the Peugue} CEMAGREF is involved in the reasearch program of the GARIH (Regional group for hydrological research), devoted to the pollution fluxes resulting from rain-water, and the means to reduce them. Cemagref is studying the Peugue, a suburban little stream near Bordeaux. The aim of this work is to characterize the river in situ, so as to specify the impacts of suburban pollutions linked with rainy periods on the environment, and to assess the effects of the forthcoming urban planning. Physico-chemical parameters and specific pollutants (heavy metals...) are measured on monthly spot samples from 11 stations all along the $6 \mathrm{~km}$ of the streamlet. Two of these stations are equipped with automatic samplers that give hourly samples to study the dynamics of pollutants during the floods.

The comparison of the chemical analysis with the distribution of macrophytes and diatom communities should lead to demonstrate the impact of different pollutions (ponctual or diffuse) existing on the catchment area. The first investigations enabled us to locate particular pollution sources and to closely connect the results of water analysis with diatom indices commonly used in France for the assessment of water quality. 
Les premières campagnes de reconnaissance du site et les premiers prélèvements laissent supposer des pollutions de type urbain et de type organique.

C'est pourquoi, afin de caractériser l'état du cours d'eau in situ, l'effet d'aménagements existants ou à venir, ainsi que les impacts biologiques des pollutions, le Cemagref se propose en 1997 d'étudier la qualité chimique des eaux et des sédiments, mais également d'utiliser des méthodes biologiques intégratrices basées sur la bio-indication et, à plus longue échéance, sur la bio-accumulation des polluants dans les matrices végétales.

\section{D PROGRAMME 1997 DU CEMAGREF}

\subsection{Etudes de la qualité chimique des eaux}

Deux types de prélèvements sont prévus en liaison avec les caractéristiques du réseau pluvial arrivant dans le Peugue :

- des tournées systématiques et régulières de prélèvement d'échantillons d'eau sur un ensemble de 11 stations. Ces échantillons seront analysés selon les paramètres classiques de qualité d'eau, tout en recherchant la présence de molécules écotoxiques ;

- des prélèvements et analyses d'eau en périodes de crues sur un premier site : le Burck. Cette station a été retenue en raison de sa position : étant le plus en aval, elle subit en effet l'accumulation de toutes les pollutions survenant le long du Peugue (utilisation d'échantillonneur automatique).

Dans un deuxième temps, des recherches seront conduites pour remonter à l'exutoire de ces pollutions, soit par appareillage installé au préalable, soit par tournées de prélèvements correctement positionnées en fonction des épisodes de crues.

\subsection{Etudes de bio-indication}

Ces études seront basées sur l'observation des macrophytes aquatiques et des diatomées fixées. Le but est de caractériser les impacts de pollutions, soit chroniques, soit lors de pluies, sur l'hydrosystème.

\subsubsection{Les macrophytes aquatiques}

L'utilisation des macrophytes comme descripteurs de la qualité des eaux et/ou des habitats n'est pas une idée récente: les premiers travaux dans ce domaine sont ceux de Butcher (1933) en Grande Bretagne.

Depuis, des travaux typologiques ou phytosociologiques ont été entrepris par divers auteurs européens, débouchant sur des méthodes opérationnelles en matière d'indices macrophytes. La plupart de ces méthodes restent cependant difficiles à généraliser. En effet, si ces travaux indiquent bien que des relations fortes existent entre les populations de macrophytes et la qualité des eaux, ils restent souvent trop adaptés à un contexte régional ou local pour être directement applicables.

Les questions principales qui continuent de se poser dans ce domaine ont trait à la nature même de la bio-indication des macrophytes. Ils intègrent les conditions physiques du milieu et la qualité des eaux dans des proportions qu'il sera probablement difficile de préciser, aussi semble-t-il nécessaire d'analyser leur capacité de bio-indicateurs de manière globale, vis-à-vis de la qualité des milieux.

Les travaux engagés en France dans ce domaine ont récemment fait l'objet d'une proposition d'indices. Considérés comme provisoires et expérimentaux, ces indices macrophytes doivent encore être testés.
L'approche en termes de bio-indication et d'application de ces indices macrophytes au programme consacré au Peugue comportera :

- une description linéaire des habitats disponibles pour les macrophytes, comprenant des observations sur les déterminants physiques de la répartition des végétaux, tels que les écoulements, les substrats, etc...

- des mesures physico-chimiques in situ et des analyses chimiques des eaux, destinées à préciser l'évolution longitudinale de la qualité des eaux,

- un inventaire des taxons de macrophytes présents sur le cours,

- une analyse des relations entre déterminants physiques et chimiques et la répartition des macrophytes, débouchant sur des précisions concernant le caractère bio-indicateur des espèces rencontrées,

- l'application des indices macrophytes en cours de test.

\subsubsection{Les diatomées fixées}

Des inventaires exhaustifs seront réalisés sur l'ensemble des stations retenues à partir des algues récoltées sur supports naturels et artificiels (carreaux de céramique immergés).

Les effets éventuels des contaminants sur ces communautés seront examinés selon diverses approches classiques :

- évolution longitudinale de la structure des communautés (diversité et richesse spécifiques),

- effets sur la densité ou la taille des diatomées (présence éventuelle de formes tératogènes,

- recherche des caractéristiques autoécologiques dominantes par compilation automatisée de bases de données de la littérature scientifique permettant de suivre l'évolution des formes polluo-résistantes, saprophiles, hétérotrophes ou halophiles, etc....

- application de divers indices diatomiques (qualité biologique, similitudes) et confrontation aux résultats des analyses physico-chimiques.

\subsection{Etudes de bio-accumulation et bio-concentration}

Les références bibliographiques traitant de bio-accumulation et de la bio-concentration de polluants dans les matrices végétales ou en relation avec la diagnose des milieux sont à l'heure actuelle peu nombreuses. Cependant, le champ d'investigation paraît tout à fait intéressant et novateur pour un repérage de pollutions par micropolluants.

L'objectif de la présente étude est de repérer des espèces végétales susceptibles de concentrer des molécules écotoxiques présentes en permanence dans les eaux, ou de manière intermittente en fonction du rythme des pluies ou des caractéristiques spécifiques d'émission des polluants sur ce bassin versant.

L'intérêt de cette méthode est le caractère intégrateur des végétaux. Ceux-ci permettraient d'appréhender des contaminations chroniques ou intermittentes des eaux que l'on ne détecte pas forcément par des analyses chimiques périodiques.

\section{III — PREMIERS RÉSULTATS - COMMEN- TAIRES}

D'après les études antérieures et les visites du début de l'année, nous avons choisi 11 stations d'observation qui nous sont apparues nécessaires pour bien localiser les nombreuses arrivées d'eau pluviale dans le Peugue (fig. l). 


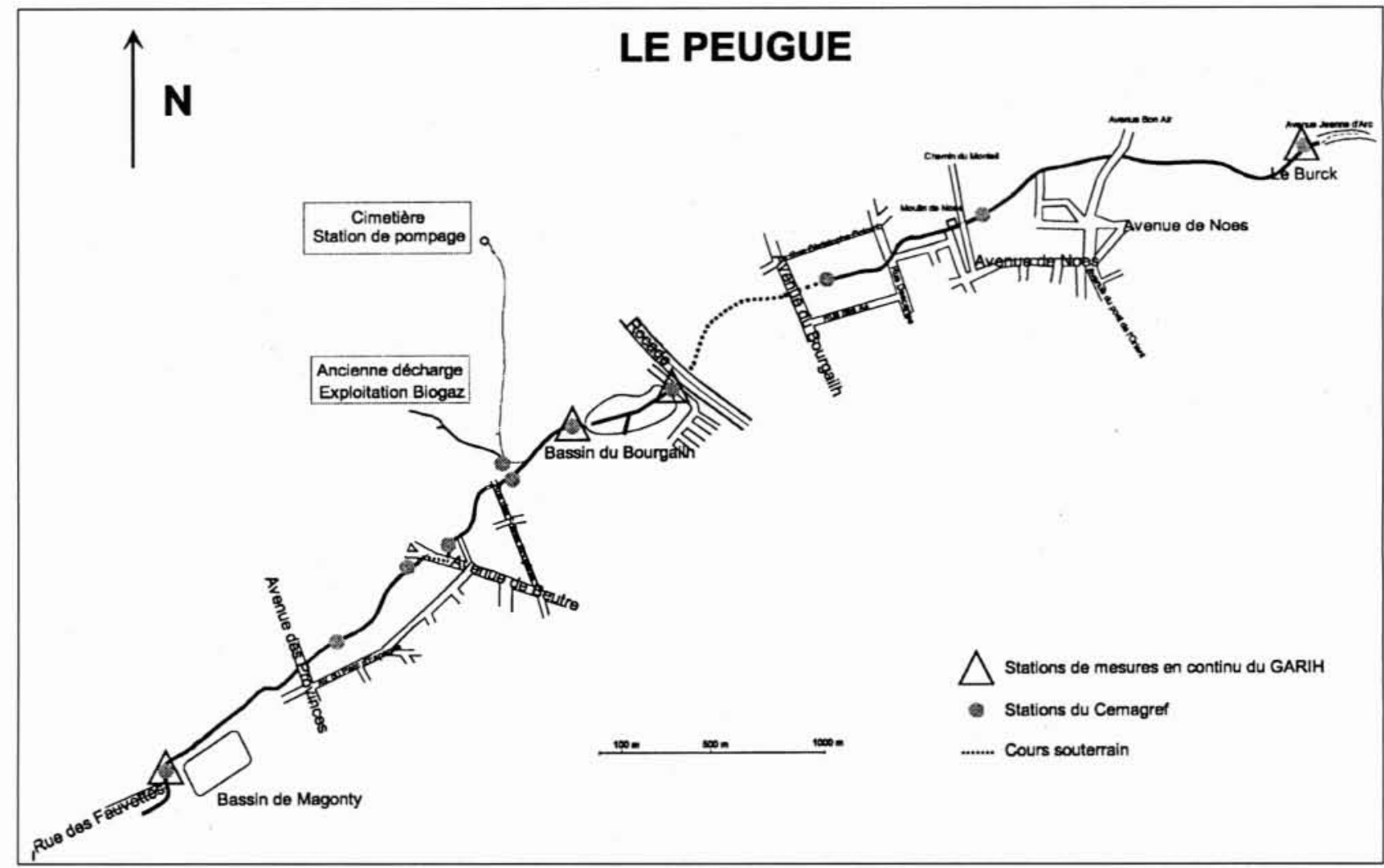

1. Cartographie du ruisseau.

\subsection{Qualité chimique de l'eau}

Une première campagne de prélèvements d'eau a été effectuée le 29 janvier 1997. Les paramètres physico-chimiques suivis sont les suivants : $\mathrm{pH}$, conductivité, température, oxygène dissous mesurés in situ, et MES, MVS, formes de l'azote $\left(\mathrm{NH}_{4}, \mathrm{NO}_{2}, \mathrm{NO}_{3}, \mathrm{NTK}\right), \mathrm{SO}_{4}, \mathrm{PO}_{4}, \mathrm{P}$ total, cations ( $\mathrm{Ca}, \mathrm{Mg}, \mathrm{Na}, \mathrm{K})$.

Si cette première campagne n'est pas suffisante pour tirer des conclusions formelles sur la qualité de l'eau du Peugue, il est toutefois intéressant de remarquer une forte dégradation entre les points 4 et 5 et ceci pour tous les paramètres, ce qui laisse supposer la présence d'un rejet polluant significatif.

Il est à noter que le dernier épisode pluvieux date du 27 janvier, soit 48 heures avant les prélèvements.
L'affluent rive gauche dont la confluence avec le Peugue se situe en amont du bassin du Bourgailh apparaît comme étant de bonne qualité et joue donc un rôle de diluant.

\subsection{Bio-indication par les diatomées}

Les premiers inventaires diatomiques réalisés à partir des substrats naturels (épilithon et périphyton), ont permis de recenser plus de 150 taxons sur les 11 stations. Cette microflore diversifiée est enrichie à l'amont du Bourgailh par des espèces plus acidophiles (Station 6).

La qualité biologique de l'eau exprimée par ces algues au travers des indices diatomiques reste néanmoins très moyenne, avec des apports préoccupants en rive droite au niveau du Bourgailh.

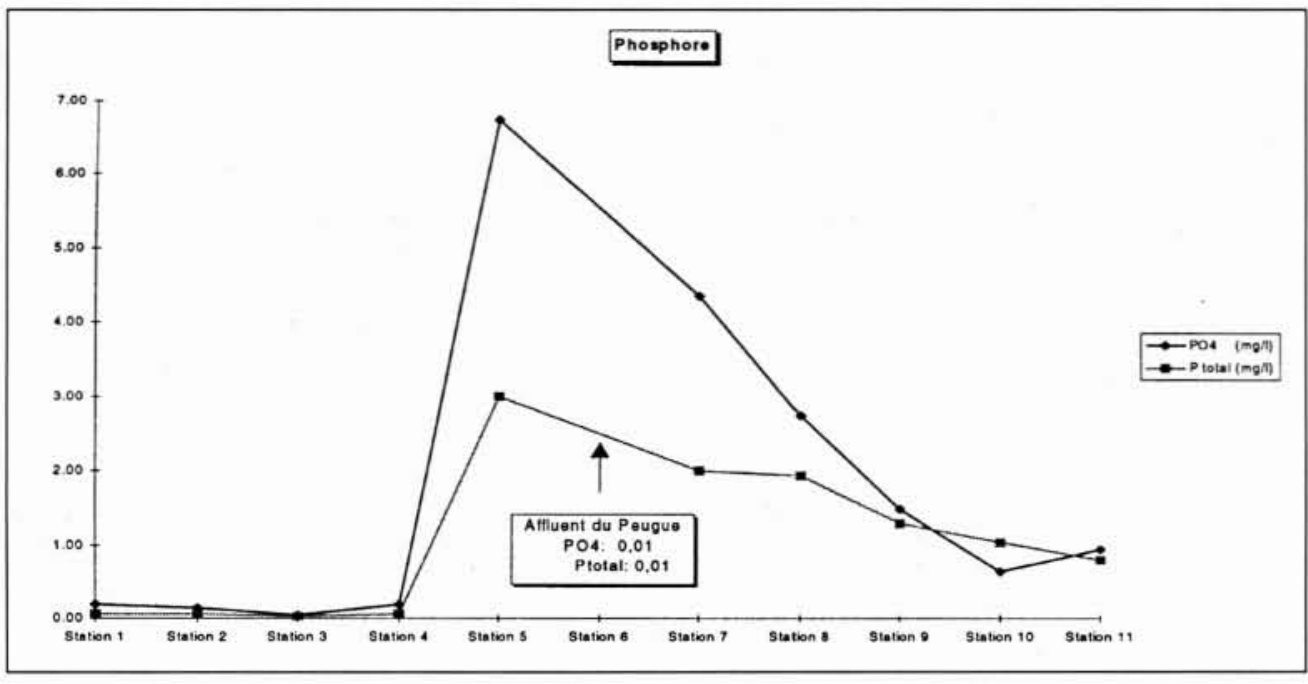

2. Exemple du phosphore. 


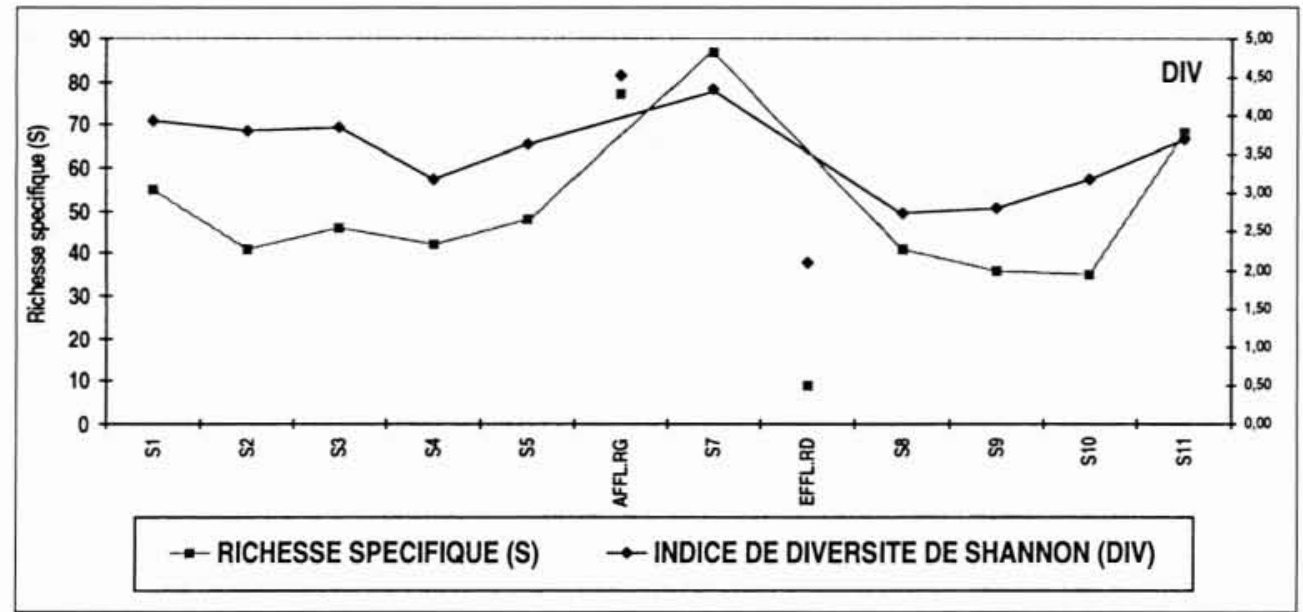

3. Evolution de la richesse et de la diversité spécifiques des diatomées du Peugue en février 1997.

4. Application de l'indice diatomique IPS aux diatomées du Peugue en février 1997 (substrats naturels).
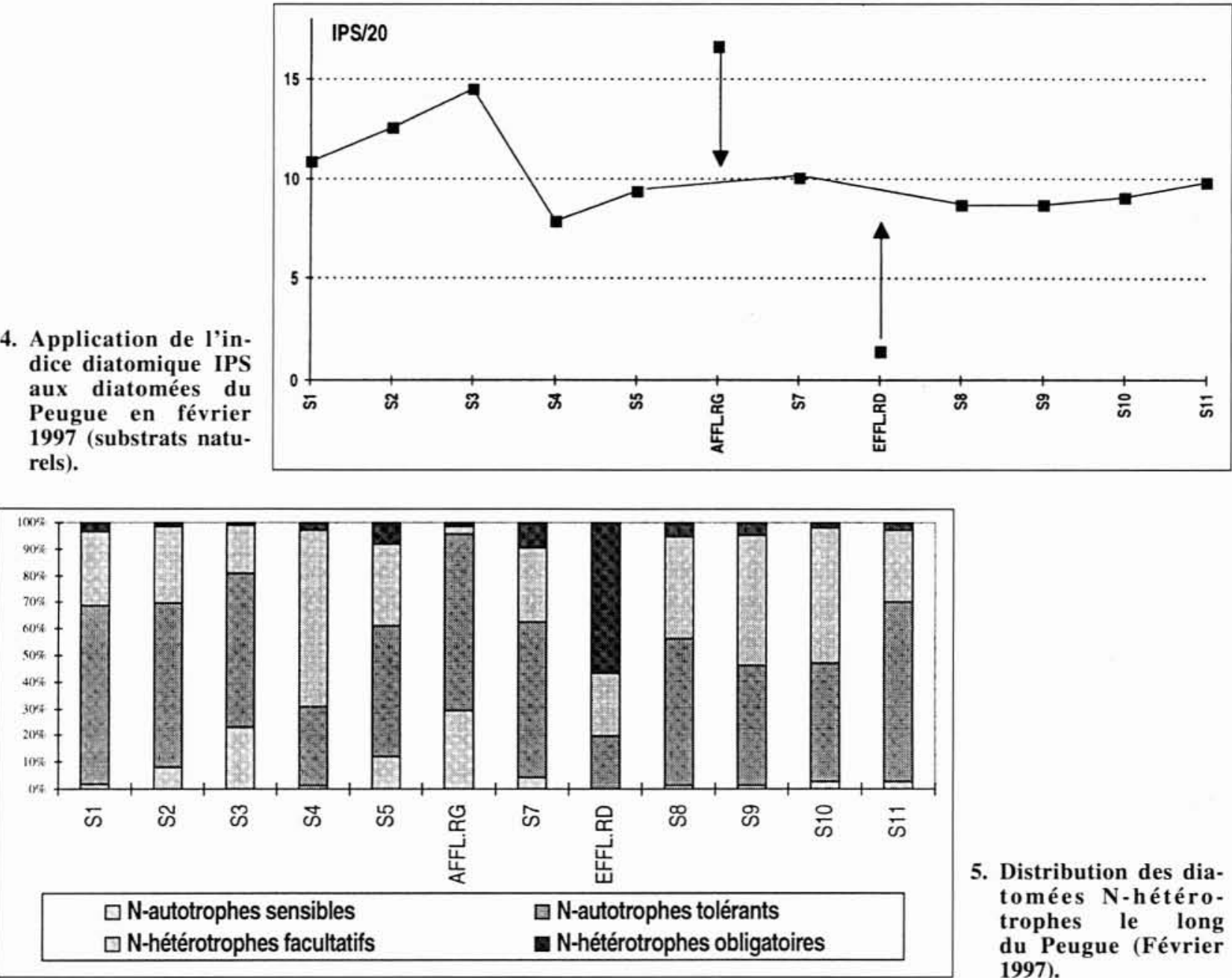

5. Distribution des diatomées N-hétérotrophes le long du Peugue (Février 1997).

L'application de l'indice IPS (Indice de Polluosensibilité Spécifique, Cemagref 1982), qui utilise tous les taxons présents, conforte les résultats de la physico-chimie pour l'affluent de rive gauche (Station 6) qui apparaît d'excellente qualité.

La chute d'indice observée à la station 4 (fig. 4)pourrait être imputable à la nature des substrats prospectés (feuille plastique à défaut de pierres) et montre s'il en était besoin, la nécessité d'utiliser des substrats normalisés. L'examen des caractéristiques autoécologiques confirme les résultats de l'IPS avec un accroissement notable des formes N-hétérotrophes dès la station 4.

\section{D REMARQUES FINALES}

Les premières investigations du Cemagref dans le domaine de l'évaluation des pollutions péri-urbaines sur le compartiment biologique fournissent quelques éléments de réflexion concernant l'évolution longitudinale de la qualité des eaux et du milieu. Il n'en reste pas moins que de nombreux facteurs ne sont encore ni répertoriés ni analysés, ce qui conduira à réaliser diverses campagnes d'observation, de mesure et de prélèvements pour analyses ultérieures, et une synthèse des données obtenues pour améliorer la connaissance du fonctionnement et de la qualité du ruisseau. 